\title{
The Effect of High-Frequency Parametric Excitation on a Stochastically Driven Pantograph-Catenary System
}

\author{
R. H. Huan, ${ }^{1}$ W. Q. Zhu, ${ }^{1}$ F. Ma, ${ }^{2}$ and Z. H. Liu ${ }^{3}$ \\ ${ }^{1}$ Department of Mechanics, Zhejiang University, Hangzhou 310027, China \\ ${ }^{2}$ Department of Mechanical Engineering, University of California, Berkeley, CA 94720, USA \\ ${ }^{3}$ Department of Civil Engineering, Xiamen University, Xiamen 361005, China
}

Correspondence should be addressed to Z. H. Liu; liuzh@xmu.edu.cn

Received 5 June 2013; Accepted 7 August 2013; Published 11 February 2014

Academic Editor: Reza Jazar

Copyright (C) 2014 R. H. Huan et al. This is an open access article distributed under the Creative Commons Attribution License, which permits unrestricted use, distribution, and reproduction in any medium, provided the original work is properly cited.

\begin{abstract}
In high-speed electric trains, a pantograph is mounted on the roof of the train to collect power through contact with an overhead catenary wire. The effect of fast harmonic and parametric excitation on a stochastically driven pantograph-catenary system is studied in this paper. A single-degree-of-freedom model of the pantograph-catenary system is adopted, wherein the stiffness of the nonlinear spring has a time-varying component characterized by both low and high frequencies. Using perturbation and harmonic averaging, a Fokker-Planck-Kolmogorov equation governing the stationary response of the pantograph-catenary system is set up. Based on the transition probability density of the stationary response, it is found that even small high-frequency parametric excitation has an appreciable effect on the system response. Among other things, it shifts the resonant frequency and often changes the response characteristics markedly.
\end{abstract}

\section{Introduction}

A number of important structures can be modeled as a stochastically driven nonlinear system subjected to both slow and fast harmonic and parametric excitations. An example is the pantograph-catenary system in railway engineering. High-speed electric trains often employ a pantograph to collect their currents from an overhead catenary system. During operation, the pantograph is excited by forces due to train-body vibration, ambient air flow, contact wire irregularities, and other disturbances. These disturbances can be realistically taken as a stochastic excitation to the pantograph. Owing to the stiffness variation between the support poles and the short-distance droppers, the catenary may be regarded as a nonlinear spring with a time-varying stiffness component. As a result, the combined pantographcatenary system is an example of a stochastically driven nonlinear system with both low- and high-frequency parametric excitations.

There is fairly extensive literature on the dynamics of pantograph-catenary systems [1-6]. However, previous studies have focused on deterministic excitations. It has been accepted that parametrically induced vibration of a pantograph-catenary system occurs mainly in the lowfrequency region. Thus earlier studies usually ignored the high-frequency parametric effect generated by the catenary $[7,8]$. Recent theoretical studies on dynamical systems, however, suggest that high-frequency parametric excitation could shift the resonant frequency or equilibrium states [912], thus altering the stability [13-15] and other response characteristics [16].

In this work, the effect of fast harmonic and parametric excitation on a stochastically driven pantograph-catenary system is investigated. A nonlinear single-degree-of-freedom model of the pantograph-catenary system possessing lowand high-frequency time-varying stiffness is adopted. Using perturbation, an approximate equation governing only the low-frequency motion is derived. Then, an averaging method based on harmonic functions [17-21] is applied to the low-frequency equation. Subsequently, a Fokker-PlanckKolmogorov equation governing the stationary response of the pantograph-catenary system is set up. Based on the transition probability density of the stationary response, the 


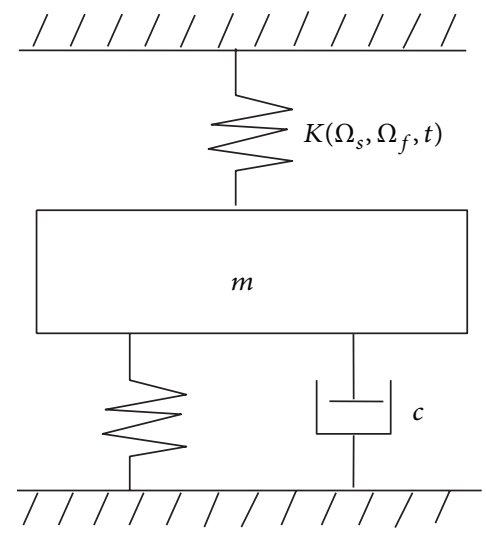

FIGURE 1: Dynamic model of pantograph-catenary system.

effect of fast parametric excitation on the resonant frequency and the primary resonant response are studied. Finally, direct numerical simulations of the nonlinear model are performed to validate the analysis presented.

\section{Model of Pantograph-Catenary System}

A commonly used model in railway engineering of the pantograph-catenary system $[3,7,8]$ is the single-degreeof-freedom model shown in Figure 1. Since the stiffness variation is repeated in each span of the catenary, the catenary stiffness possesses periodic components. If we consider the stiffness variation between the vertical droppers, the catenary is usually taken as a spring with time-varying stiffness components given by $[1,7,8]$

$$
K\left(\Omega_{s}, \Omega_{f}, t\right)=K_{0}(V)\left(1+\alpha \cos \left(\Omega_{s} t\right)+\beta \cos \left(\Omega_{f} t\right)\right),
$$

where $V$ represents the speed of train and $K_{0}(V)$ is the speeddependent average stiffness of the catenary. In the above equation, $\alpha, \beta$ are unspecified coefficients and $\alpha \cos \left(\Omega_{s} t\right)$, $\beta \cos \left(\Omega_{f} t\right)$ account for the stiffness fluctuations between the support poles and the vertical droppers, respectively. Let $L_{s}$ and $L_{d}$ denote, respectively, the span distance and dropper distance. Then the frequencies of stiffness fluctuation can be expressed as

$$
\Omega_{s}=\frac{2 \pi V}{L_{s}} ; \quad \Omega_{f}=\frac{2 \pi V}{L_{d}} .
$$

In general, $L_{s} \gg L_{d}$ and therefore $\Omega_{f} \gg \Omega_{s}$. In many cases, the average stiffness $K_{0}(V)$ can be approximated by a quadratic function of the train speed [8] such that

$$
K_{0}(V)=K_{s}-K_{d} V^{2}
$$

where $K_{s}$ is the static average stiffness of the catenary and $K_{d}$ is a coefficient accounting for dynamic interactions of the coupled pantograph-catenary system. The value of $K_{s}$ can be calculated by the finite element method.

Due to random disturbances (such as train body movement, contact wire irregularity, and ambient air flow), a weak random excitation $c_{w} \xi(t)$ is introduced into the equation of the pantograph-catenary system. Incorporating stiffness nonlinearity, the equation of motion of the pantographcatenary system can be written as

$$
\begin{aligned}
m \ddot{y}+ & c \dot{y}+k^{\prime} y+\widetilde{k} y^{3}+\left(K_{s}-K_{d} V^{2}\right) \\
& \times\left(1+\alpha \cos \left(\Omega_{s} t\right)+\beta \cos \left(\Omega_{f} t\right)\right) y=c_{w} \xi(t),
\end{aligned}
$$

where $y$ is the vertical displacement of the pantograph system and the fourth item in the left of (4) represents the interaction of pantograph and catenary system.

Let $\omega_{n}^{2}=\bar{k} / m$ and $\tau=\omega_{n} t$ where $\bar{k}=k^{\prime}+K_{s}-K_{d} V^{2}$ and $\tau$ is a nondimensional time. Equation (4) can be converted into the nondimensional form

$$
\begin{aligned}
& y^{\prime \prime}+2 \zeta y^{\prime}+y+b y^{3} \\
&= {\left[-E_{11}+E_{12} r_{s}^{2}\right] \cos \left(r_{s} \tau\right) y } \\
&+\left[-E_{21}+E_{22} r_{f}^{2}\right] \cos \left(r_{f} \tau\right) y+h_{0} \xi(\tau),
\end{aligned}
$$

where $y^{\prime}=d y / d \tau, y^{\prime \prime}=d^{2} y / d \tau^{2}$, and

$$
\begin{gathered}
2 \zeta=\frac{c}{m \omega_{n}}, \quad b=\frac{\tilde{k}}{m \omega_{n}^{2}}, \quad E_{11}=\frac{\alpha K_{s}}{m \omega_{n}^{2}}, \\
E_{12}=\frac{\alpha K_{d}}{m}\left(\frac{L_{s}}{2 \pi}\right)^{2}, \quad E_{21}=\frac{\beta K_{s}}{m \omega_{n}^{2}}, \\
E_{22}=\frac{\beta K_{d}}{m}\left(\frac{L_{d}}{2 \pi}\right)^{2}, \quad h_{0}=\frac{c_{w}}{m \omega_{n}^{2}}, \\
r_{s}=\frac{\Omega_{s}}{\omega_{n}}=\frac{2 \pi V}{\omega_{n} L_{s}}, \quad r_{f}=\frac{\Omega_{f}}{\omega_{n}}=\frac{2 \pi V}{\omega_{n} L_{d}} .
\end{gathered}
$$

Here $r_{s}=O(1)$ is the nondimensional low-frequency excitation, and $r_{f} \gg 1$ is the nondimensional high-frequency excitation. If a train is travelling at a constant speed $V$, then $2 \zeta, E_{11}, E_{12} r_{s}^{2}, E_{21}, E_{22} r_{f}^{2}$, and $h_{0}$ are all small constants. Suppose these constants are of the same order in a small parameter $\varepsilon$. System (5) is a randomly excited Duffing oscillator with both slow and fast time-varying stiffness. The goal of the present work is to investigate the influence of the fast parametric excitation on the characteristics of system (5).

\section{Approximate Equation Governing the Slow Motion}

Introduce two different time-scales:

$$
T_{0}=r_{f} \tau=\varepsilon^{-1} \tau ; \quad T_{1}=\tau,
$$

where the slow time $T_{1}$ and the fast time $T_{0}$ are considered as new independent variables in (5). Separate $y(\tau)$ into a slow part $w\left(T_{1}\right)$ and a fast part $\varepsilon \phi\left(T_{0}, T_{1}\right)[10]$ so that

$$
y(\tau)=w\left(T_{1}\right)+\varepsilon \varphi\left(T_{0}, T_{1}\right)
$$


It has been recognized that the behavior of system (5) is mainly described by the slow part $w$ since $\varepsilon \phi$ is small compared to $w$. Let $\langle\cdot\rangle_{T_{0}}=\left(\int_{0}^{2 \pi} \cdot d T_{0}\right) / 2 \pi$ be the time-averaging operator over one period of the fast time scale $T_{0}$ with the slow time $T_{1}$ fixed. Assume that $\varepsilon \phi$ and its derivatives vanish upon $T_{0}$-averaging so that $\langle y(\tau)\rangle_{T_{0}}=$ $w\left(T_{1}\right)$. Substitute (8) into (5) to obtain

$$
\begin{aligned}
D_{1}^{2} w & +\left(\varepsilon D_{1}^{2}+2 D_{0} D_{1}+\varepsilon^{-1} D_{0}^{2}\right) \varphi+2 \zeta\left(D_{1} w+D_{0} \varphi+\varepsilon D_{1} \varphi\right) \\
& +(w+\varepsilon \varphi)+b(w+\varepsilon \varphi)^{3} \\
= & {\left[-E_{11}+E_{12} r_{s}^{2}\right](w+\varepsilon \varphi) \cos \left(r_{s} T_{1}\right) } \\
& \times\left[-E_{21}+\varepsilon^{-1} E_{22} r_{f}\right](w+\varepsilon \varphi) \cos \left(T_{0}\right)+h_{0} \xi\left(T_{1}\right),
\end{aligned}
$$

where $D_{i}^{j}=\partial^{j} / \partial T_{i}^{j} \quad(i=0,1 ; j=1,2)$. Average equation (9) with respect to $T_{0}$ and subtract the averaged equation from (9); an approximate expression for $\phi$ is obtained by considering only the dominant terms of order $\varepsilon^{-1}$ as

$$
D_{0}^{2} \varphi=E_{22} r_{f} w \cos \left(T_{0}\right) .
$$

The stationary solution to first order for $\phi$ is

$$
\varphi=-E_{22} r_{f} w \cos \left(T_{0}\right) .
$$

Substitute (11) into (9) and apply $T_{0}$-averaging. Retain dominant terms of order $\varepsilon^{0}$ to obtain

$$
\begin{aligned}
D_{1}^{2} w & +2 \zeta D_{1} w+\left[1+\frac{\left(E_{22} r_{f}\right)^{2}}{2}\right] w+b w^{3} \\
& =\left[-E_{11}+E_{12} r_{s}^{2}\right] w \cos \left(r_{s} T_{1}\right)+h_{0} \xi\left(T_{1}\right) .
\end{aligned}
$$

Equation (12) governs only the slow motion $w$ of system (5). Note that the fast excitation affects the slow behavior of system (12) by adding $\left(E_{22} r_{f}\right)^{2} / 2$ to the linear stiffness. By numerical simulations, the probability densities $p(a)$ of the amplitude of the original system (5) and of the slow system (12) are plotted in Figure 2. It is observed that the larger the fast excitation parameter $E_{22} \cdot r_{f}$, the bigger the difference between the two amplitudes.

\section{Effect of Fast Parametric Excitation}

In the last section, an approximate equation governing only the slow motion of system (5) is obtained by perturbation. In the following, we will discuss the effect of the fast harmonic excitation on this slow system in greater detail.

4.1. Effect on Resonant Frequency. Let $\omega^{2}=\left[1+\left(E_{22} r_{f}\right)^{2} / 2\right]$. In order to study the effect of the fast parametric excitation on the resonant frequency of system (12), we will first consider the free response of system (12) governed by

$$
D_{1}^{2} w+\omega^{2} w+b w^{3}=0 .
$$

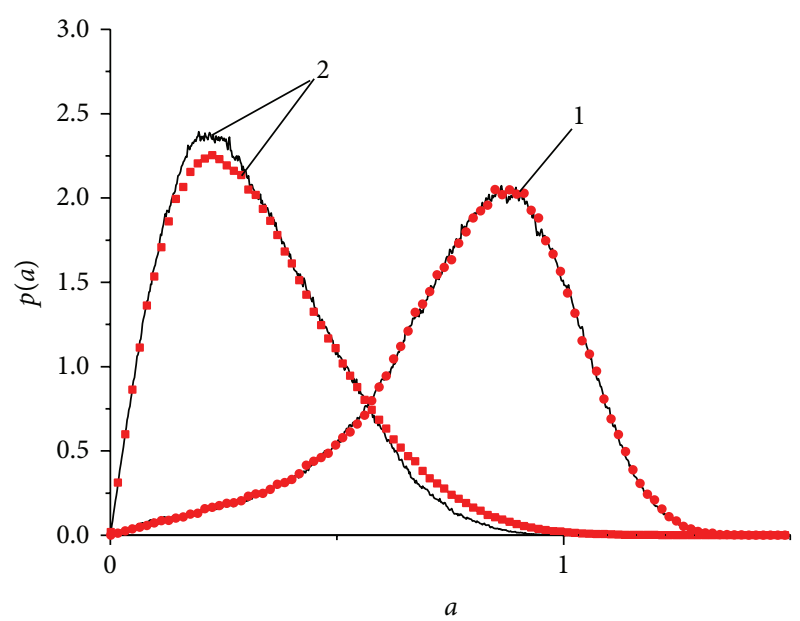

Figure 2: Probability densities of amplitude of the original system (5) and of the slow system (12). Line 1: no fast excitation; line 2: $E_{22}$. $r_{f}=0.8$. Solid lines for the original system (5) and dotted lines for the slow system (12).

The periodic solution of system (13) has the form [17]

$$
\begin{aligned}
& w\left(T_{1}\right)=a \cos \theta\left(T_{1}\right), \quad D_{1} w\left(T_{1}\right)=-a \nu(a, \theta) \sin \theta\left(T_{1}\right), \\
& \theta\left(T_{1}\right)=\phi\left(T_{1}\right)+\gamma,
\end{aligned}
$$

where $a$ is the amplitude, $\gamma$ is the phase angle, and

$$
\begin{gathered}
\nu(a, \theta)=\frac{d \phi}{d T_{1}}=\sqrt{\left(\omega^{2}+\frac{3 b a^{2}}{4}\right)(1+\eta \cos (2 \theta))}, \\
\eta=\frac{\left(b a^{2} / 4\right)}{\left(\omega^{2}+3 b a^{2} / 4\right)} .
\end{gathered}
$$

The instantaneous frequency $v(a, \theta)$ can be approximated by the finite sum:

$$
v(a, \theta)=b_{0}(a)+b_{2}(a) \cos 2 \theta+b_{4}(a) \cos 4 \theta+b_{6}(a) \cos 6 \theta,
$$

where

$$
\begin{gathered}
b_{0}(a)=\sqrt{\left(\omega^{2}+\frac{3 b a^{2}}{4}\right)}\left(1-\frac{\eta^{2}}{16}\right) \\
b_{2}(a)=\sqrt{\left(\omega^{2}+\frac{3 b a^{2}}{4}\right)}\left(\frac{\eta}{2}+\frac{3 \eta^{3}}{64}\right) \\
b_{4}(a)=\sqrt{\left(\omega^{2}+\frac{3 b a^{2}}{4}\right)}\left(-\frac{\eta^{2}}{16}\right) \\
b_{6}(a)=\sqrt{\left(\omega^{2}+\frac{3 b a^{2}}{4}\right)}\left(\frac{\eta^{3}}{64}\right) .
\end{gathered}
$$




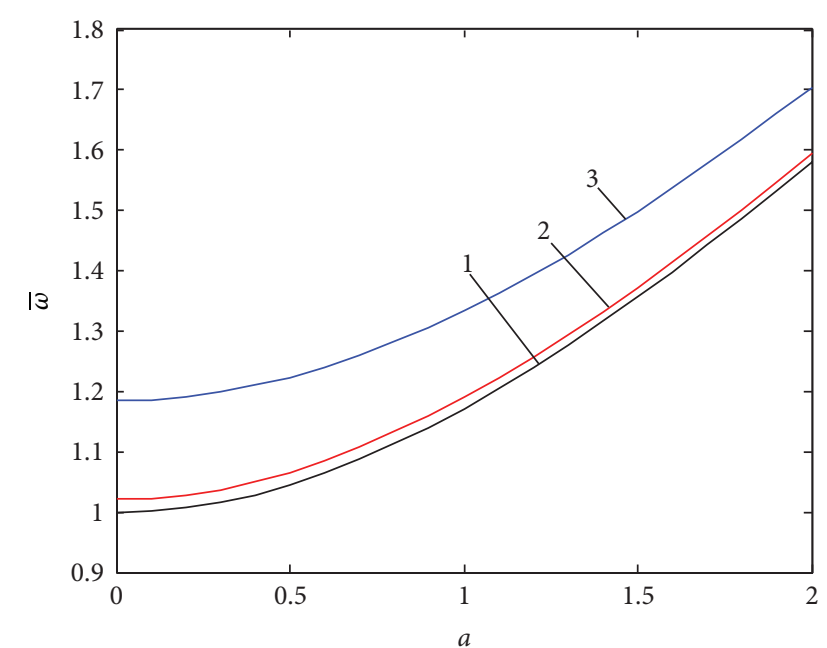

FIGURE 3: Average resonant frequency for different values of the fast excitation parameter $E_{22} \cdot r_{f}$. Line 1: no fast excitation; line 2: $E_{22} \cdot r_{f}$ $=0.3$; line $3: E_{22} \cdot r_{f}=0.9$.

By integrating (16) with respect to $\theta$ from 0 to $2 \pi$, an average frequency

$$
\bar{\omega}(a)=\frac{1}{2 \pi} \int_{0}^{2 \pi} \nu(a, \theta) d \theta=b_{0}(a)
$$

of the oscillator is obtained. The approximate relation

$$
\theta\left(T_{1}\right) \approx \bar{\omega}(a) T_{1}+\gamma
$$

will be used in the averaging process that follows. Note that the resonant frequency $v(a, \theta)$ depends on both the amplitude $a$ and phase $\gamma$. In Figure 3, the average resonant frequency $\bar{\omega}(a)$ is plotted against the amplitude $a$ for different values of $E_{22} \cdot r_{f}$. As the fast excitation parameter $E_{22} \cdot r_{f}$ increases, the average resonant frequency of the system also increases.

4.2. Effect on Resonant Response. We now proceed to examine the effect of fast parametric excitation on the resonant response of system (12). It is reasonable to assume that neither light damping nor a weak random excitation will destabilize system (12). In this case the response of system (12) can be regarded as a random spread of the periodic solutions of system (13). As a consequence,

$$
\begin{gathered}
w\left(T_{1}\right)=A \cos \Theta\left(T_{1}\right), \\
D_{1} w\left(T_{1}\right)=-A V(A, \Theta) \sin \Theta\left(T_{1}\right), \\
\Theta\left(T_{1}\right)=\Phi\left(T_{1}\right)+\Gamma\left(T_{1}\right),
\end{gathered}
$$

where $A, \Theta, \Phi, \Gamma$, and $V$ are all random processes. The instantaneous and average frequencies of system (12) are of the same forms given by (15) and (18).
Substitute (20) into (12) and treat (20) as generalized van der Pol transformation from $w, D_{1} w$ to $A, \Gamma$; the following equations for $A$ and $\Gamma$ can be obtained:

$$
\begin{aligned}
& \frac{d A}{d T_{1}}=F_{1}\left(A, \Theta, r_{s} T_{1}\right)+G_{1}(A, \Theta) \xi\left(T_{1}\right), \\
& \frac{d \Gamma}{d T_{1}}=F_{2}\left(A, \Theta, r_{s} T_{1}\right)+G_{2}(A, \Theta) \xi\left(T_{1}\right),
\end{aligned}
$$

where

$$
\begin{aligned}
& F_{1}\left(A, \Theta, r_{s} T_{1}\right) \\
& =-\frac{1}{\omega^{2}+b A^{2}}(2 \zeta A V(A, \Theta) \sin \Theta \\
& \left.\quad+\left[-E_{11}+E_{12} r_{s}^{2}\right] A \cos \Theta \cos \left(r_{s} T_{1}\right)\right) \\
& \quad \times V(A, \Theta) \sin \Theta, \\
& \left.F_{2}\left(A, \Theta, r_{s} T_{1}\right) \quad+\left[-E_{11}+E_{12} r_{s}^{2}\right] A \cos \Theta \cos \left(r_{s} T_{1}\right)\right) \\
& =-\frac{1}{\omega^{2} A+b A^{3}}(2 \zeta A V(A, \Theta) \sin \Theta \\
& \times V(A, \Theta) \cos \Theta \\
& G_{1}(A, \Theta)=-\frac{h_{0}}{\omega^{2}+b A^{2}} V(A, \Theta) \sin \Theta \\
& \quad G_{2}(A, \Theta)=-\frac{h_{0}}{\omega^{2} A+b A^{3}} V(A, \Theta) \cos \Theta,
\end{aligned}
$$

and $h_{0}$ has been specified in (6).

4.2.1. The Case with $h_{0}=0$. Firstly, we consider the pure parametric harmonic excitation case. Neglect the diffusion terms and (21) can be rewritten as

$$
\begin{aligned}
\frac{d A}{d T_{1}}= & -\frac{1}{\omega^{2}+b A^{2}} \\
& \times(2 \zeta A V(A, \Theta) \sin \Theta \\
& \left.+\left[-E_{11}+E_{12} r_{s}^{2}\right] A \cos \Theta \cos \left(r_{s} T_{1}\right)\right) \\
& \times V(A, \Theta) \sin \Theta \\
\frac{d \Gamma}{d T_{1}}= & -\frac{1}{\omega^{2} A+b A^{3}} \\
& \times(2 \zeta A V(A, \Theta) \sin \Theta \\
& \left.+\left[-E_{11}+E_{12} r_{s}^{2}\right] A \cos \Theta \cos \left(r_{s} T_{1}\right)\right) \\
& \times V(A, \Theta) \cos \Theta .
\end{aligned}
$$

The nonlinear system (23) is subjected to harmonic parametric excitations and there is the possibility of parametric 
resonance. Since large response of the pantograph-catenary system may cause malfunctions in power collection, we will emphasize the primary parametric resonance case. Assume that in primary parametric resonance there exists

$$
\frac{r_{s}}{\bar{\omega}(A)}=2+\varepsilon \eta
$$

where $\bar{\omega}(A)$ is the average frequency of system (12) and $\varepsilon \eta \ll$ 1 is the small detuning parameter. Multiply (24) by $T_{1}$ and utilize the approximate relation (19) to obtain

$$
r_{s} T_{1}=2 \Theta+\varepsilon \eta \bar{\omega} T_{1}-2 \Gamma .
$$

Introduce a new variable $\Delta=\varepsilon \eta \bar{\omega} T_{1}-2 \Gamma$ so that (25) can be rewritten as

$$
r_{s} T_{1}=2 \Theta+\Delta
$$

Substitute (26) into (23) and average (23) with respect to the rapidly varying process $\Theta$ from 0 to $2 \pi$ to generate the following averaged differential equations:

$$
\begin{aligned}
\frac{d A}{d T_{1}}=( & -A\left[512 \zeta \omega^{2}+320 \zeta b A^{2}\right. \\
& \left.\left.-64\left(2 b_{0}-b_{4}\right)\left(-E_{11}+E_{12} r_{s}^{2}\right) \sin \Delta\right]\right) \\
\times( & \left.512\left(\omega^{2}+b A^{2}\right)\right)^{-1} \\
\frac{d \Delta}{d T_{1}}=r_{s}- & 2 b_{0}+\frac{\left(2 b_{0}+2 b_{2}+b_{4}\right)\left(-E_{11}+E_{12} r_{s}^{2}\right) \cos \Delta}{4\left(\omega^{2}+b A^{2}\right)} .
\end{aligned}
$$

Equation (27) involves only slowly varying processes $A$ and $\Delta$. By letting $d A / d T_{1}=d \Delta / d T_{1}=0,(27)$ gives the frequency response relation:

$$
\begin{aligned}
& {\left[\frac{512 \zeta \omega^{2} A+320 \zeta b A^{3}}{64 A\left(2 b_{0}-b_{4}\right)\left(-E_{11}+E_{12} r_{s}^{2}\right)}\right]^{2}} \\
& \quad+\left[\frac{4\left(r_{s}-2 b_{0}\right)\left(\omega^{2}+b A^{2}\right)}{\left(2 b_{0}+2 b_{2}+b_{4}\right)\left(-E_{11}+E_{12} r_{s}^{2}\right)}\right]^{2}=1 .
\end{aligned}
$$

Numerical results are obtained for $\zeta=0.1, b=0.5, E_{11}=1.2$, $E_{12}=0.1$, and $E_{21}=0.5$ and shown in Figures 4 and 5. Figure 4 displays the frequency response under primary parametric resonance for different values of the fast excitation parameter $E_{22} \cdot r_{f}$. It is observed that fast parametric excitation shifts the resonant peaks to the right, which means that a higher frequency of the slow excitation is required to produce the resonant response. The dependence of the amplitude $\Delta a$ at $r_{s}=2.0$ and the width of the resonant region $\Delta \Omega$ as a function of the fast excitation parameter $E_{22} \cdot r_{f}$ is shown in Figure 5. The amplitude and the width of the resonant region are reduced appreciably by fast parametric excitation.

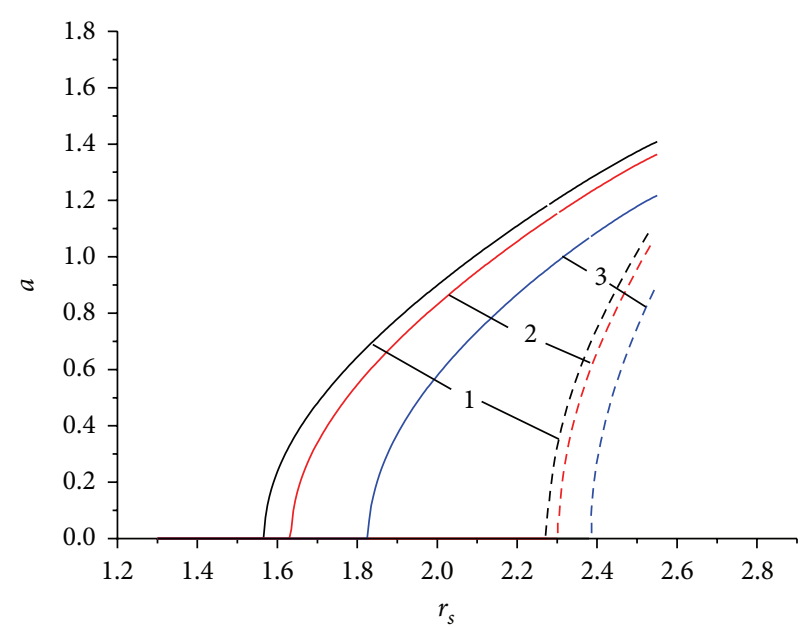

FIGURE 4: Frequency response for primary parametric resonance. Dashed lines are unstable. Line 1: no fast excitation; line 2: $E_{22} \cdot r_{f}=$ 0.3 ; line $3: E_{22} \cdot r_{f}=0.6$.

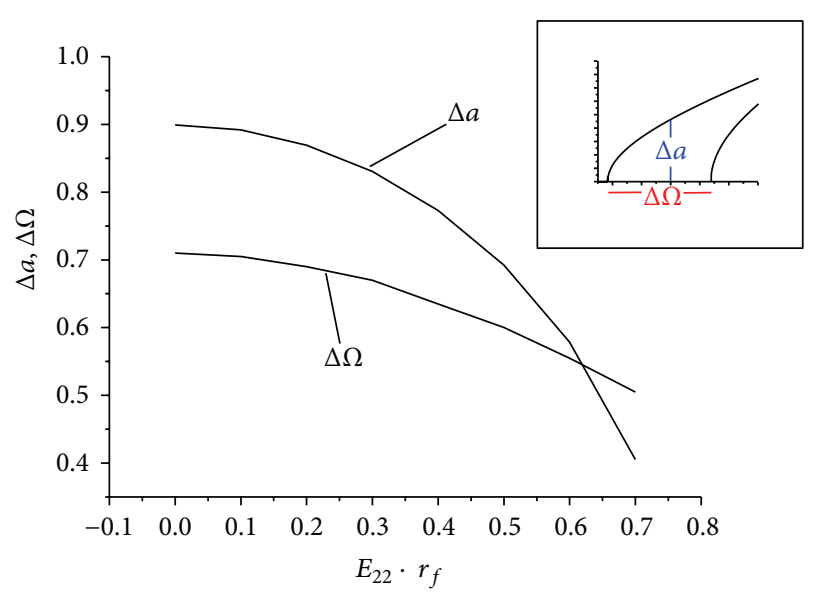

FIgURE 5: The amplitude $\Delta a$ at $r_{s}=2.0$ and the width of the resonant region $\Delta \Omega$ as functions of the fast excitation parameter $E_{22} \cdot r_{f}$.

4.2.2. The Case with $h_{0} \neq 0$. In practical railway engineering, random disturbances are always present and the term $\xi\left(T_{1}\right)$ cannot be neglected. Suppose that the stochastic excitation $\xi\left(T_{1}\right)$ is a weak Gaussian white noise with intensity $2 D$. Then, (21) can be modeled as Stratonovich stochastic differential equations and transformed into the following Itô equations by adding Wong-Zakai correction terms [18]:

$$
\begin{aligned}
& d A=m_{1}\left(A, \Theta, r_{s} T_{1}\right) d T_{1}+\sigma_{1}(A, \Theta) d B\left(T_{1}\right), \\
& d \Gamma=m_{2}\left(A, \Theta, r_{s} T_{1}\right) d T_{1}+\sigma_{2}(A, \Theta) d B\left(T_{1}\right),
\end{aligned}
$$

where $d B\left(T_{1}\right)$ is the unit Wiener process and

$$
\begin{aligned}
& m_{1}\left(A, \Theta, r_{s} T_{1}\right)=F_{1}\left(A, \Theta, r_{s} T_{1}\right)+D\left(G_{1} \frac{\partial G_{1}}{\partial A}+G_{2} \frac{\partial G_{1}}{\partial \Theta}\right), \\
& m_{2}\left(A, \Theta, r_{s} T_{1}\right)=F_{2}\left(A, \Theta, r_{s} T_{1}\right)+D\left(G_{1} \frac{\partial G_{2}}{\partial A}+G_{2} \frac{\partial G_{2}}{\partial \Theta}\right),
\end{aligned}
$$




$$
\begin{aligned}
& b_{1}(A, \Theta)=\sigma_{1}^{2}(A, \Theta)=2 D G_{1}^{2}, \\
& b_{2}(A, \Theta)=\sigma_{2}^{2}(A, \Theta)=2 D G_{2}^{2},
\end{aligned}
$$

and $F_{i}$ and $G_{i}(i=1,2)$ are given in (22). In primary parametric resonance, utilize (26) and average the rapidly varying process $\Theta$ from 0 to $2 \pi$ to generate the averaged Itô stochastic differential equations:

$$
\begin{aligned}
& d A=\bar{m}_{1}(A, \Delta) d T_{1}+\bar{\sigma}_{1}(A) d B\left(T_{1}\right), \\
& d \Delta=\bar{m}_{2}(A, \Delta) d T_{1}+\bar{\sigma}_{2}(A) d B\left(T_{1}\right),
\end{aligned}
$$

where the averaged drift and diffusion coefficients are

$$
\begin{aligned}
\bar{m}_{1}(A, \Delta)=( & A\left[512 \zeta \omega^{2}+320 \zeta b A^{2}\right. \\
& \left.\left.-64\left(2 b_{0}-b_{4}\right)\left(-E_{11}+E_{12} r_{s}^{2}\right) \sin \Delta\right]\right) \\
& \times\left(512\left(\omega^{2}+b A^{2}\right)\right)^{-1} \\
+ & \frac{h_{0}^{2} D\left(4 b^{2} A^{2}+12 b \omega^{2} A^{2}+32 \omega^{4}\right)}{64 A\left(\omega^{2}+b A^{2}\right)^{3}}, \\
\bar{m}_{2}(A, \Delta)= & r_{s}-2 b_{0} \\
& +\frac{\left(2 b_{0}+2 b_{2}+b_{4}\right)\left(-E_{11}+E_{12} r_{s}^{2}\right) \cos \Delta}{4\left(\omega^{2}+b A^{2}\right)}, \\
\bar{b}_{1}(A)= & \bar{\sigma}_{1}^{2}(A)=\frac{h_{0}^{2} D\left(16 \omega^{2}+10 b A^{2}\right)}{16\left(\omega^{2}+b A^{2}\right)^{2}}, \\
\bar{b}_{2}(A)= & \bar{\sigma}_{2}^{2}(A)=\frac{h_{0}^{2} D\left(16 \omega^{2}+14 b A^{2}\right)}{16 A^{2}\left(\omega^{2}+b A^{2}\right)^{2}} .
\end{aligned}
$$

The Fokker-Planck-Kolmogorov (FPK) equation associated with the Itô equations (31) is

$$
\begin{aligned}
\frac{\partial p}{\partial T_{1}}= & -\frac{\partial}{\partial a}\left(\bar{m}_{1} p\right)-\frac{\partial}{\partial \Delta}\left(\bar{m}_{2} p\right) \\
& +\frac{1}{2} \frac{\partial^{2}}{\partial a^{2}}\left(\bar{b}_{1} p\right)+\frac{1}{2} \frac{\partial^{2}}{\partial \Delta^{2}}\left(\bar{b}_{2} p\right),
\end{aligned}
$$

where $p=p\left(a, \Delta, T_{1}\right)$ is the probability density of amplitude $a$ and phase $\Delta$. The initial condition for (33) is

$$
p=\delta\left(a-a_{0}\right) \delta\left(\Delta-\Delta_{0}\right), \quad T_{1}=0,
$$

and the boundary conditions for (33) are

$$
\begin{gathered}
p=\text { finite, } \quad a=0, \\
p, \frac{\partial p}{\partial a} \longrightarrow 0, \quad a \longrightarrow \infty, \\
p\left(a, \Delta+2 n \pi, T_{1} \mid a_{0}, \Delta_{0}, T_{10}\right)=p\left(a, \Delta, T_{1} \mid a_{0}, \Delta_{0}, T_{10}\right) .
\end{gathered}
$$

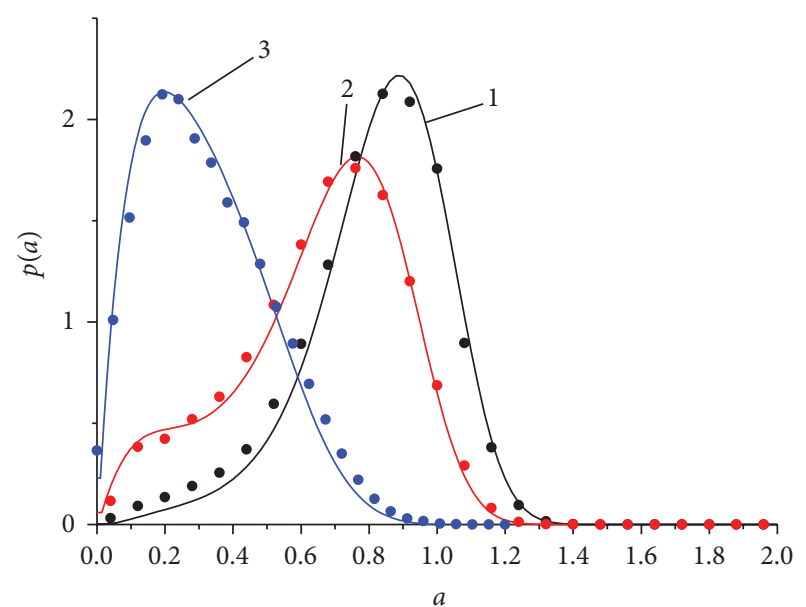

FIGURE 6: Stationary probability density $p(a)$ for different values of the fast excitation parameter $E_{22} \cdot r_{f}$. Line 1: no fast excitation; line 2: $E_{22} \cdot r_{f}=0.4$; line 3: $E_{22} \cdot r_{f}=0.8$. Dotted lines are from direct numerical simulations of the nonlinear model (5).

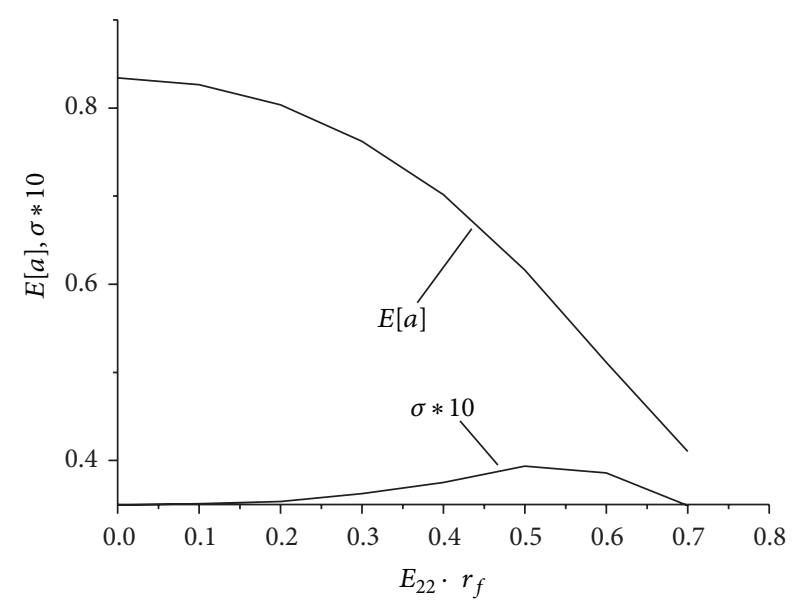

FIgURE 7: Mean and variance of the amplitude of the slow system (12).

The nonlinear three-dimensional parabolic problem as given in (33)-(35) does not admit an easy solution, analytically or numerically. Fortunately, in practical applications we are more interested in the stationary solution of the FPK equation (33). In this case, (33) can be simplified by letting $\partial p / \partial \tau=$ 0 . Then, the joint stationary probability density $p(a, \Delta)$ is obtained readily by using the finite difference method. The stationary probability density of the amplitude $p(a)$ can be obtained from $p(a, \Delta)$ by

$$
p(a)=\int_{0}^{2 \pi} p(a, \Delta) d \Delta .
$$

Numerical results for $p(a)$ and $p(a, \Delta)$ of system (12) in parametric resonance are obtained for $\zeta=0.1, b=0.5$, $E_{11}=1.2, E_{12}=0.1, E_{21}=0.5, r_{s}=2.0, D=1.0$, $h_{0}=0.1$, and shown in Figures 6-8. Figure 6 shows the stationary probability density $p(a)$ for different values of the fast excitation parameter $E_{22} \cdot r_{f}$. Fast parametric excitation 


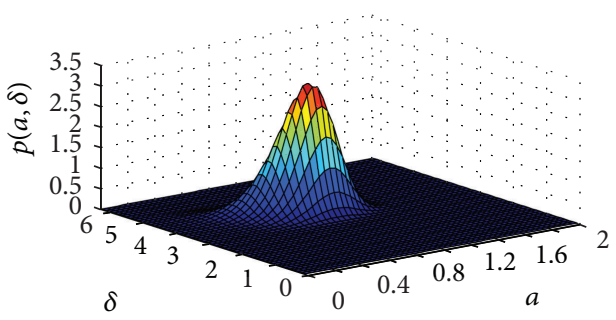

(a)

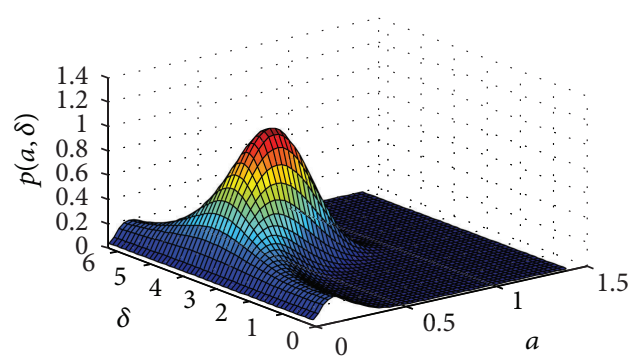

(b)

FIGURE 8: Joint stationary probability density $p(a, \Delta)$. (a): No fast excitation; (b) $E_{22} \cdot r_{f}=0.8$.

shifts the probability density curve to the left, changing both the peak height and shape. Even when the fast excitation is small, the response of the slow system (12) may change dramatically. This observation is reinforced in Figure 7, in which the mean and variance of the amplitude $a$ of system (12) change significantly upon adding fast parametric excitation. This reflects the increased stiffness of the slow system (12) under fast excitation. Finally, direct numerical simulations of the nonlinear model (5) are performed to generate $p(a)$. As shown in Figure 6, data from direct numerical simulations closely match those generated by (36), thus validating the analysis presented. The joint probability density $p(a, \Delta)$ of the slow system (12) is plotted in Figure 8.

\section{Conclusions}

In the present paper, the effect of fast parametric excitation on a stochastically excited pantograph-catenary system has been investigated. A nonlinear model of the pantographcatenary system has been adopted, wherein the stiffness of the nonlinear spring has a time-varying component characterized by both low and high frequencies. The overall parametrically induced motion of the system is separated into two parts: a dominant low-frequency vibration which is the main motion and a small high-frequency vibration which affects the low-frequency motion by altering the stiffness. Using perturbation, an approximate equation governing only the low-frequency motion has been derived. An averaging method for harmonic functions has been applied to obtain the primary resonant response of the low-frequency motion.

Analytical results show that the effect of fast parametric excitation is not negligible. The addition of even a small amount of high-frequency parametric excitation may dramatically increase the resonant frequency and change the primary resonant response of a system. From a theoretical viewpoint, an investigation of a Duffing oscillator subjected to both stochastic and parametric forces has been conducted to study the surprising effect of high-frequency input. Practically speaking, many structures outside railway engineering can be modeled as a stochastically driven nonlinear system excited by both slow and fast parametric excitations. Hence, the results of this investigation could be useful in other applications.

\section{Conflict of Interests}

The authors declare that there is no conflict of interests regarding the publication of this paper.

\section{Acknowledgments}

The authors gratefully acknowledge the financial supblackport provided by the Natural Science Foundation of China (nos. 10932009 and 11372271), 973 Program (no. 2011CB711105), National Key Technology Support Program (no. 2009BAG12A01) and Natural Science Foundation of Zhejiang Province (no. LY12A02004). Opinions, findings, and conclusions expressed in this paper are those of the authors and do not necessarily reflect the views of the sponsors.

\section{References}

[1] G. Galeotti and P. Toni, "Nonlinear modeling of a railway pantograph for high speed running," Transactions on Modelling and Simulation, vol. 5, pp. 421-436, 1993.

[2] P. H. Poznic, J. Jerrelind, and L. Drugge, "Experimental evaluation of nonlinear dynamics and coupled motions in a pantograph," in Proceedings of the ASME International Design Engineering Technical Conferences and Computers and Information in Engineering Conference (DETC '09), pp. 619-626, San Diego, Calif, USA, September 2009.

[3] L. Drugge, T. Larsson, A. Berghuvud, and A. Stensson, "The nonlinear behavior of a pantograph current collector suspension," in Proceedings of the ASME Design Engineering Technical Conferences, pp. 1-7, Las Vegas, Nev, USA, 1999.

[4] S. Levy, J. A. Bain, and E. J. Leclerc, "Railway overhead contact systems, catenary-pantograph dynamics for power collection at high speeds," Journal of Engineering for Industry, vol. 90, no. 4, pp. 692-699, 1968.

[5] J.-W. Kim, H.-C. Chae, B.-S. Park, S.-Y. Lee, C.-S. Han, and J.H. Jang, "State sensitivity analysis of the pantograph system for a high-speed rail vehicle considering span length and static uplift force," Journal of Sound and Vibration, vol. 303, no. 3-5, pp. 405427, 2007.

[6] G. Poetsch, J. Evans, R. Meisinger et al., "Pantograph/catenary dynamics and control," Vehicle System Dynamics, vol. 28, no. 23, pp. 159-195, 1997. 
[7] T. X. Wu and M. J. Brennan, "Basic analytical study of pantograph-catenary system dynamics," Vehicle System Dynamics, vol. 30, no. 6, pp. 443-456, 1998.

[8] T. X. Wu and M. J. Brennan, "Dynamic stiffness of a railway overhead wire system and its effect on pantograph-catenary system dynamics," Journal of Sound and Vibration, vol. 219, no. 3, pp. 483-502, 1999.

[9] D. Tcherniak and J. J. Thomsen, "Slow effects of fast harmonic excitation for elastic structures," Nonlinear Dynamics, vol. 17, no. 3, pp. 227-246, 1998.

[10] R. Bourkha and M. Belhaq, "Effect of fast harmonic excitation on a self-excited motion in van der Pol oscillator," Chaos, Solitons \& Fractals, vol. 34, no. 2, pp. 621-627, 2007.

[11] J. J. Thomsen, "Some general effects of strong high-frequency excitation: stiffening, biasing and smoothening," Journal of Sound and Vibration, vol. 253, no. 4, pp. 807-831, 2002.

[12] J. J. Thomsen, "Using fast vibrations to quench friction-induced oscillations," Journal of Sound and Vibration, vol. 228, no. 5, pp. 1079-1102, 1999.

[13] J. S. Jensen, "Non-linear dynamics of the follower-loaded double pendulum with added support-excitation," Journal of Sound and Vibration, vol. 215, no. 1, pp. 125-142, 1998.

[14] J. S. Jensen, "Quasi-static equilibria of a buckled beam with added high-frequency excitation," DCAMM Report, Technical University of Denmark, Copenhagen, Denmark, 1998.

[15] J. S. Jensen, "Pipes conveying fluid pulsating with high frequency," DCAMM Report no. 563, Technical University of Denmark, Copenhagen, Denmark, 1998.

[16] E. P. Popov and I. P. Paltov, Approximate Methods for Analyzing Nonlinear Automatic Systems, Fizmatgiz, Moscow, Russia, 1960.

[17] Z. Xu and Y. K. Cheung, "Averaging method using generalized harmonic functions for strongly non-linear oscillators," Journal of Sound and Vibration, vol. 174, no. 4, pp. 563-576, 1994.

[18] Z. L. Huang and W. Q. Zhu, "Averaging method for quasiintegrable Hamiltonian systems," Journal of Sound and Vibration, vol. 284, no. 1-2, pp. 325-341, 2005.

[19] Z. L. Huang, W. Q. Zhu, and Y. Suzuki, "Stochastic averaging of strongly non-linear oscillators under combined harmonic and white-noise excitations," Journal of Sound and Vibration, vol. 238, no. 2, pp. 233-256, 2000.

[20] G. O. Cai and Y. K. Lin, "Nonlinearly damped systems under simultaneous broad-band and harmonic excitations," Nonlinear Dynamics, vol. 6, no. 2, pp. 163-177, 1994.

[21] R. Haiwu, X. Wei, M. Guang, and F. Tong, "Response of a Duffing oscillator to combined deterministic harmonic and random excitation," Journal of Sound and Vibration, vol. 242, no. 2, pp. 362-368, 2001. 

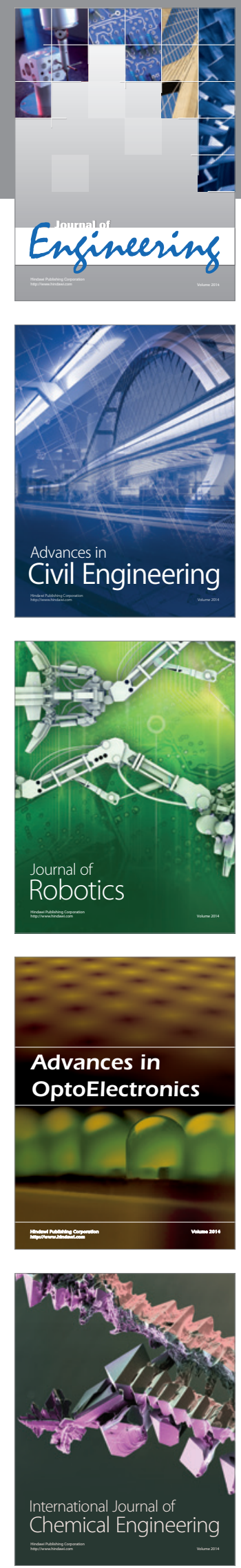

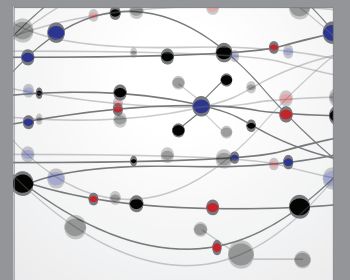

The Scientific World Journal
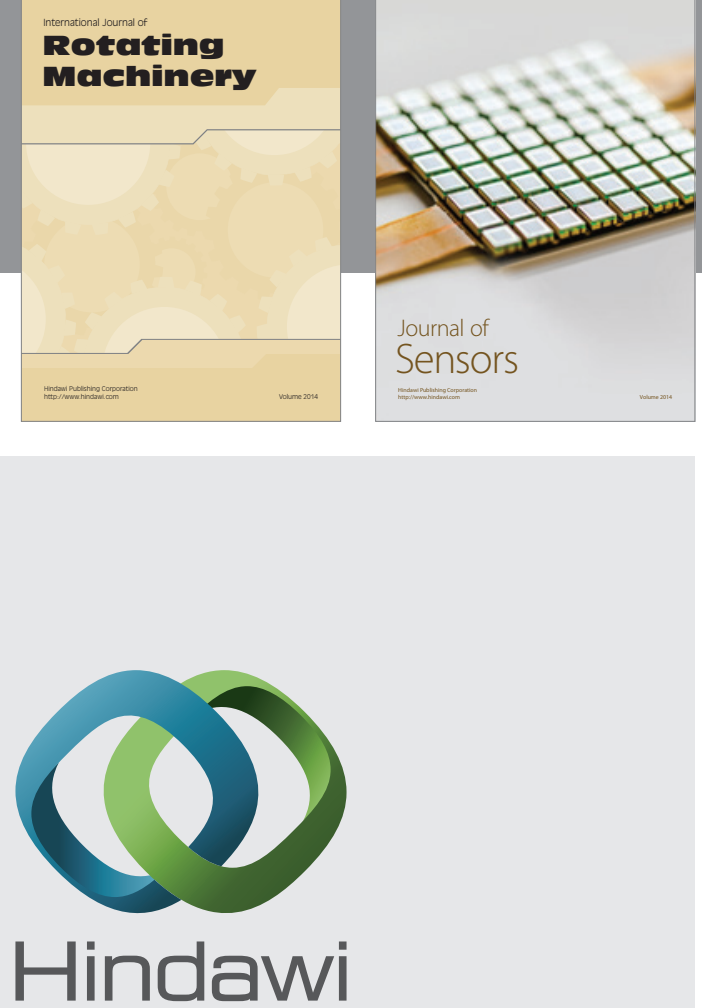

Submit your manuscripts at http://www.hindawi.com
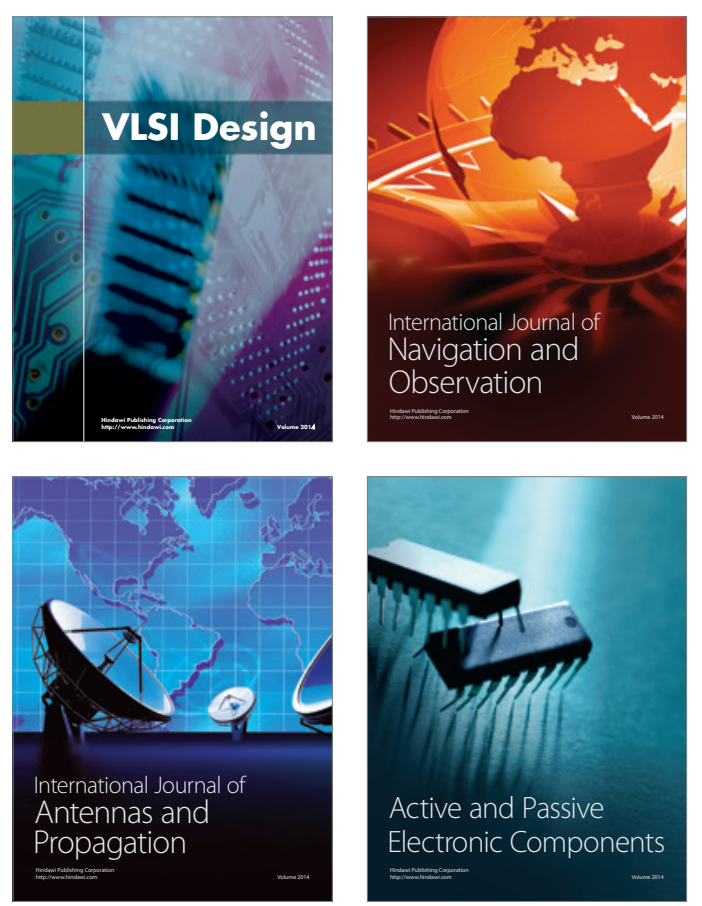
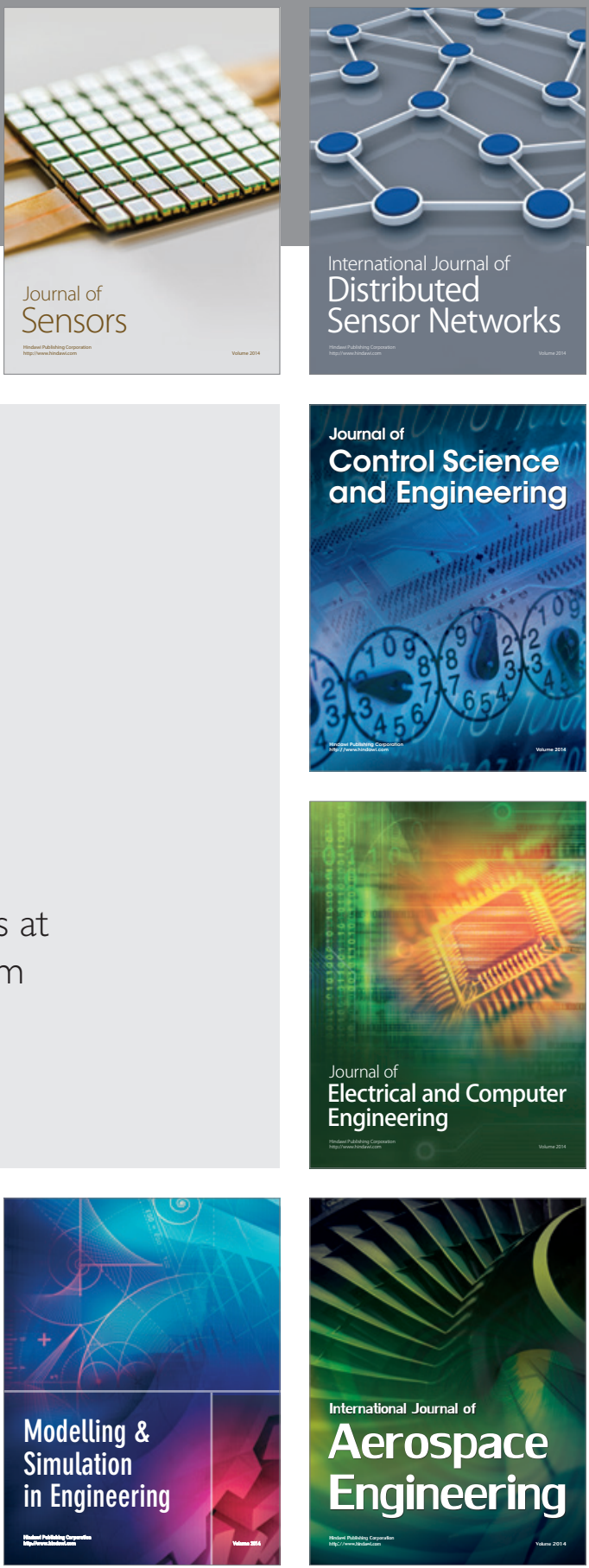

Journal of

Control Science

and Engineering
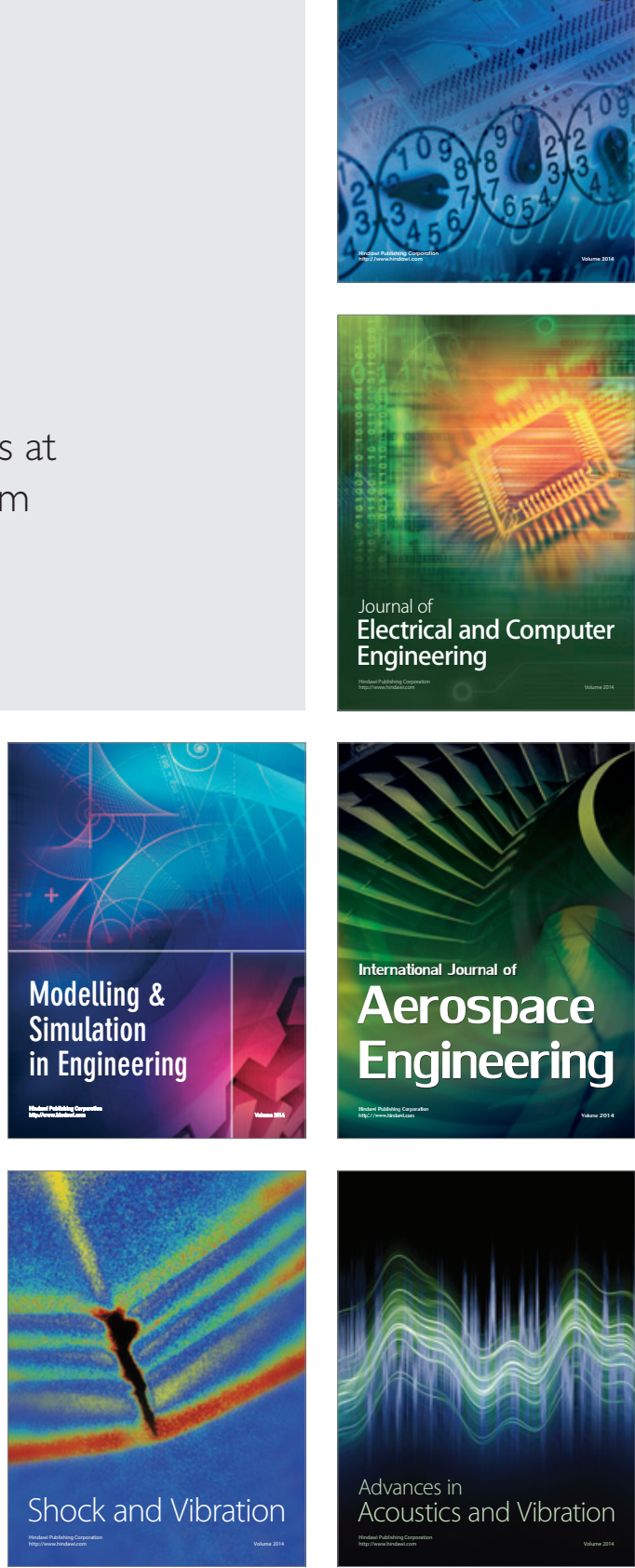